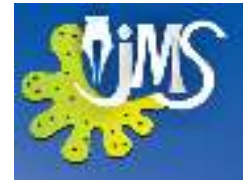

\title{
Efficient Office Assistant Robot System: Autonomous Navigation and Controlling based on ROS
}

\author{
Diddeniya S. I. A. P. ${ }^{1}$, Wanniarachchi W. K. I. L. , De Silva P. R. S. ${ }^{2}$ and Ganegoda N.C. ${ }^{3}$ \\ ${ }^{1}$ Department of Physics, University of Sri Jayewardenepura, Nugegoda, Sri Lanka \\ ${ }^{2}$ Department of Computer Sciences, University of Sri Jayewardenepura, Nugegoda, Sri Lanka \\ ${ }^{3}$ Department of Mathematics, University of Sri Jayewardenepura, Nugegoda, Sri Lanka
}

\begin{abstract}
This paper proposes a movable robot that can be used to deliver documents or small parcels $(>2.0 \mathrm{~kg})$ between employees of an unstructured indoor office environment. The study reviews literature on robot navigation, apply promising techniques and test their accuracy in an unstructured indoor laboratory environment. There are many technologies available in robot navigation. We have selected free and open source software and inexpensive sensors and materials to build a low cost but accurate robot. Our system comprised of three units: first one that process sensor data, second one which is the navigating robot having sensors and actuators, and third one controlling part of office assistant robot system. We have used Robotic Operating System (ROS) indigo as the ground operating system in the background of Ubuntu Operating System. The experiment was conducted to examine the capabilities of the setup and studied its behavior, mapping and localization specifically according to the user commands. An unstructured indoor environment was selected with five users located on the sides. We considered both dark (55 lux - 60 lux) and bright (15 lux - 20 lux) illumination conditions in the room. Then, we allowed the robot to navigate autonomously according to the user commands between five users and monitored its accuracy by looking at the completion of the route to a target user. Results show that the robot has about $98.4 \%$ of accuracy of achieving the goal location on average in both lighting conditions and $98 \%$ and $98.8 \%$ of accuracies for bright and dark conditions respectively. Finally, this office assistant robot system could be practically used in an office environment in both bright and dark lighting conditions.
\end{abstract}

KEYWORDS: Indoor robot navigation, Robotic operating system, Robot controlling, environment mapping, robot localization 


\section{INTRODUCTION}

As robots help people to do their tasks easily, they have become an interesting part in world nowadays. There are several types of robots. Some robot types need to move place to place in order to complete their tasks. Therefore, robot navigation is a very important part of robotics.

Humans could navigate in between known two places autonomously. Here they use their eyes as vision sensors, legs as actuators and brain as the processor. In human autonomous navigation, the environment is captured by human eyes, and human brain identifies the path according to the captured environment data to move to the correct location. A vision based robot autonomous navigation system imitates the human autonomous navigation. In an autonomous navigation system, the robot has a vision sensor work as eyes, movable robot base with controllable actuators that represent legs or wheels and processing unit that acts as the human brain.

In our research work, we implemented a low cost autonomously navigable robot for an indoor office environment. The system could be developed using existing computers and it requires no expensive devices for implementation. It can deliver a document or a small parcel in between two office members autonomously. In this proposed system we used iRobot Create 2 Programmable robot base which is a low cost robot base, Primesense 3D sensor as the vision sensor and a Laptop Computer as the Processing unit of the robot and a Personal Computer as the Centre Location PC.

Another important part of this research is robot controlling system. It is a userfriendly Graphical User Interface (GUI) program which can be installed on the existing computer regarding each office member. It can be used to move office assistant robot system in between office members using simple commands. Users can deliver a document or a small parcel $(>2 \mathrm{~kg}$ ) by controlling the robot using this program.

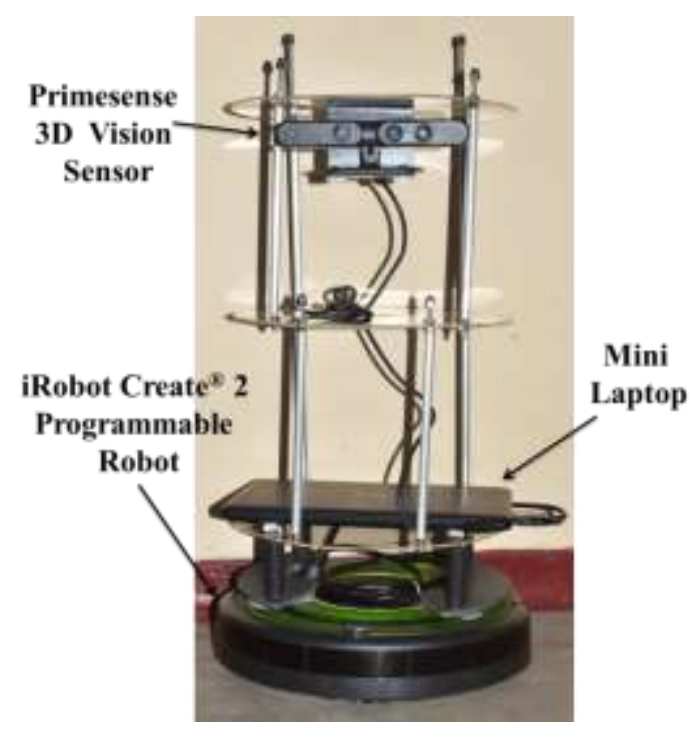

Figure 1. Structure of the Office Assistant Robot System

\section{LITERATURE SURVEY}

A robot consists of a base, which occupies actuators such as wheels or machinery legs. Among various commercially available robot bases, Kuboki hexa-base (Hamzeh and Elnagar, 2015), Pioneer 3DX (Zaman et al., 2011)and iRobot (Diddeniya et al., 2017) are mostly used in robotic research. Safdar Zaman et al have developed a ROS-based Mapping, Localization and Autonomous Navigation system using a Pioneer 3-DX Robot (Zaman et al., 2011). Another research was conducted

using Kobuki by Osama Hamzeh and Ashraf Elnagar (Hamzeh and Elnagar, 2015). However above bases are expensive compared to iRobot Create 2. Hence it has been used in our own 
previous research. There we have integrated iRobot-Arduino-Kinect Systems to build an Educational Voice and Keyboard Operated Wireless Mobile Robot (Diddeniya et al., 2017).

A movable robot needs sensors to capture the environment in order to navigate in an unknown area. In earlier researches Sonar sensors were used for mapping and navigation (Elfes and Automation, 1987). In their study, Tyrone Sherwin et.al proposes a novel approach of dynamic target localization using a single RF emitter. Through the use of multiple directional antennae, Received Signal Strength (RSS) was compared to determine the most probable direction of the targeted emitter, which is combined with the distance estimates to improve the localization performance (Sherwin et al., 2018).

Vision sensors such as RGB cameras are being used commonly (Zhou et al., 2003) but complex computations were performed for accurate results. Kinect and Primesense are 3D vision sensors that provide RGB-D data in real time. 3D vision sensors have been used for various researches including Non-Contact Human Body Parameter Measurement for virtual dressing room (Adikari et al., 2017) and for Human Computer Interaction System for Impaired People by using Kinect Motion Sensor: for capturing Voice and Gesture in Smart Home system (Rathnayake et al., 2016, Rathnayake et al., 2018). Kinect sensor has many drawbacks such as, heavy weight, large size, and need for additional power supply which affects the mobility of the robot. Further, it is not compatible with Turtlebot robotic software package (Diddeniya et al., 2017). In contrast Primesense is light weight, small in size and there is no need for an additional power supply. Specifically, it is compatible with Turtlebot robotic software package.

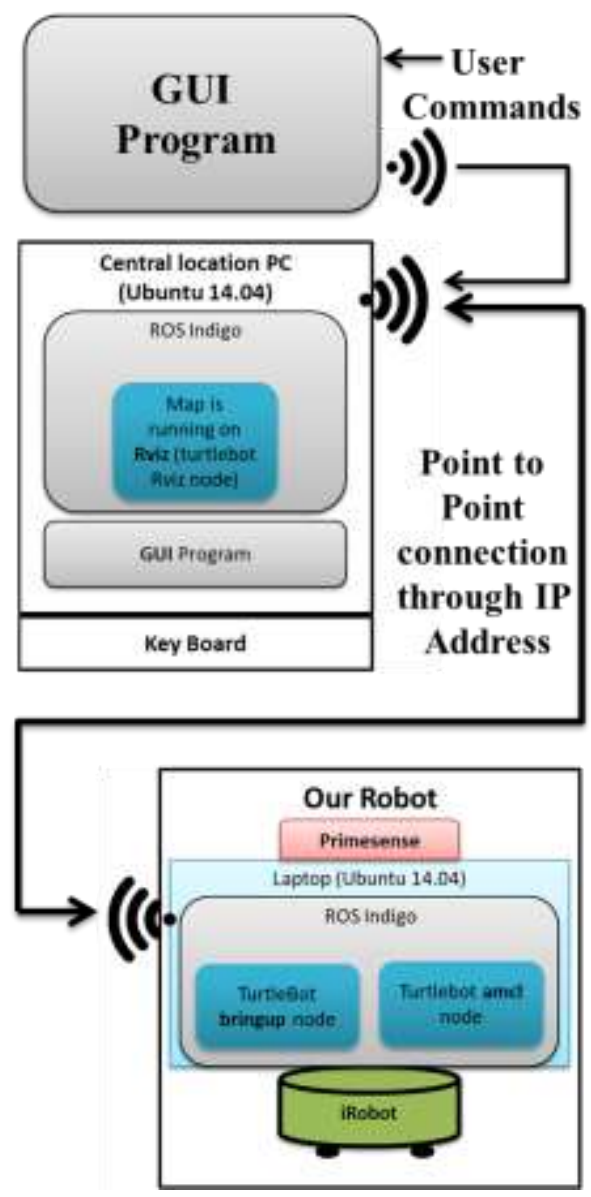

Figure 2. Office Assistant Robot System Overview

Using the data captured by the sensors, the robot navigates around the area. Localization and mapping are key elements of autonomous robot navigation. There are many algorithms built for above purposes. Artificial Neural Network (ANN) is one of these algorithms. Diogo Santos Ortiz Correa et al studied Mobile Robots Navigation in Indoor Environments Using Kinect Sensor (Zhang, 2012). The system is composed by two parts. The first part is a reactive navigation system in which a mobile 
robot moves avoiding obstacles in environment, using the distance sensor Kinect. The second part of this system uses an ANN to recognize different configurations of the environment, for example, path ahead, left path, right path and intersections. This is a finite state machine which has limited capabilities in navigation (Correa et al., 2012).

Simultaneous Localization and Mapping (SLAM) algorithm (Whyte and Magazine, 2006) is widely used in current robotic navigation (An et al., 2016, Gao et al., 2017).

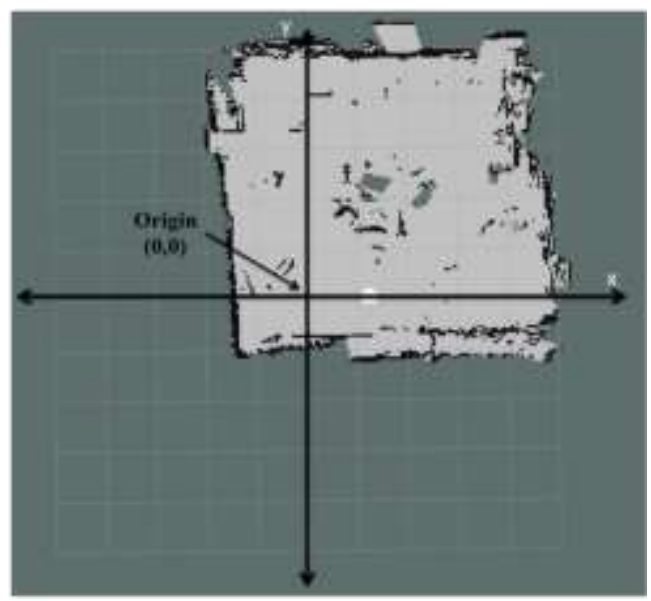

Figure 3. The generated local map of the unstructured room used for testing

Robotic Operating System (ROS) is designed for smooth building of robots. It is open source software package that works on top of Linux based Operating Systems. There are many researches carried out on ROS, making it a stable and robust system in various environments. Qunshan $\mathrm{Xu}$ et al. Designed and Implemented a ROS based Autonomous Navigation System for wheeled mobile robots (Xu et al., 2015).

A ROS-Based Indoor Autonomous Exploration and Navigation Wheelchair were built by Zhengang Li et al. The host computer program is based on the ROS, an extensible Arduino microcontroller is used to control wheelchair motors. The wheelchair adopts two-wheel differential control structure. The depth point cloud from RGB-D camera is cast to laser data and then used for map building and obstacle detection of Gmapping algorithm. The position and orientation of wheelchair are estimated by Adaptive Monte Carlo Localization (AMCL) algorithm (Li et al., 2017).

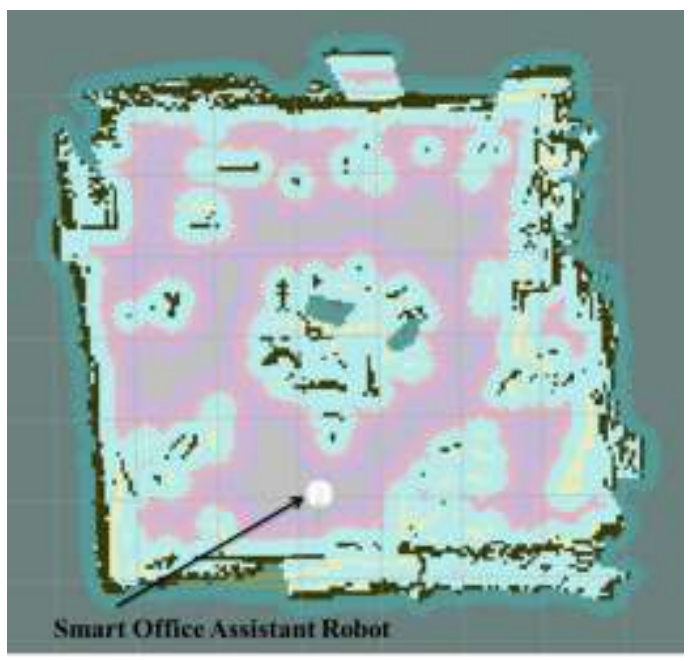

Figure 4. The real time global map of the unstructured room utilized for testing

\section{METHODOLOGY}

The structure of the robot shown in figure 1 was built using iRobot Create 2, Primesense 3D sensor and an Asus mini Laptop and a desktop computer was used as the Centre

Location PC as illustrated in figure 2. Our development environment was chosen as Ubuntu 14.04. We have installed ROS (Robotic Operating System) indigo on top of the OS. Turtlebot which is an existing package for ROS was selected as the robot kit. Among its numerous functionalities we made the use of bring 
up, gmapping, keyboard teleop, Rviz view, and amcl nodes in our study. Our experiment was conducted in an unstructured room. As in human navigation, the robot has to aware of the working environment in order to avoid collisions and to know available paths in the room. For that purpose, Robot uses a map to navigate autonomously in the environment. However, it should be first fed into the robot by humans. Following inbuilt functions of Turtlebot were used for the mapping.

\section{Building a map of unstructured room}

First, bring up, gmapping, keyboard teleop and Rviz view nodes were used to generate the map of the environment on the Centre location PC, using the environment data (RGB-D images) captured by the vision sensor mounted on the robot. While the generation of the map, the robot was controlled manually to cover the whole area of the room. The map is built on the XY Cartesian plain as shown in figure 2. To build the environment map, SLAM algorithm is used inside of gmapping node.

\section{SLAM}

SLAM is a process. A mobile robot can build a map of an environment and at the same time use this map to deduce its location using the SLAM. Both the trajectory of the platform and the location of all landmarks are estimated online without the need for any a priori knowledge of location in SLAM algorithm (Whyte and Magazine, 2006).

\section{Autonomous navigation of robot}

Then, bring up, amcl, and Rviz view nodes and the generated map were used for autonomous navigation. To initialize the robot navigation, user has to initiate a pose estimation which enables the robot to know its position on the map.

The built map is further required for localization of the autonomously navigable robot system. In the amcl node, Adaptive Monte Carlo Localization (AMCL) approach is used for localization (Hiemstra and Nederveen, 2007) and Move_Base package is used for navigation task (Chen et al., 2018).

\section{AMCL}

AMCL is a probabilistic localization method for a robot moving in 2 Dimensional environments. It implements the adaptive Monte Carlo localization (or KLD-sampling) approach, which uses a particle filter to track the pose of a robot alongside a known map.

amcl takes in a 3D vision-based map, 3D vision scans, and transform messages, and outputs pose estimates. On startup, amcl initializes its particle filter in accordance to the parameters given. If no parameters are set, the initial filter state will be a moderately sized particle cloud centred about the origin $(0,0)$, by default (Ioakeimidis, 2018).

\section{Move_Base}

The move_base node has a ROS interface for configuring, executing, and interacting with the navigation stack on a robot. Given a goal in the world, the move_base package provides an implementation of an action that, will attempt to reach it with a mobile base. The move_base node links a global and local planner together to accomplish its global navigation task. It supports any global planner and any local planner adhering to the nav_core::BaseGlobalPlanner interface and nav_core::BaseLocalPlanner interface specified in the nav_core 
package respectively. The move_base node also maintains two cost maps each for the global planner, and the local planner that are used to achieve navigation tasks (Ioakeimidis, 2018).

TABLE 1. Predefined Coordinates of user locations

\begin{tabular}{|r|c|c|c|}
\hline No: & Users & $\begin{array}{c}\text { Coordinate } \\
(\mathbf{X})\end{array}$ & $\begin{array}{c}\text { Coordinate } \\
\text { (Y) }\end{array}$ \\
\hline 1 & User 1 & 2.24 & 0.447 \\
\hline 2 & User 2 & -0.837 & 2.96 \\
\hline 3 & User 3 & -0.495 & 1.29 \\
\hline 4 & User 4 & 3.06 & 2.1 \\
\hline 5 & User 5 & 0.616 & 3.54 \\
\hline
\end{tabular}

Further, the user has to provide a goal location using keyboard. Location is provided through a pre-assigned coordinates of user positions on map. Our robot calculates the minimum route to the goal location using map that was generated earlier. Then robot navigates to the given location. While its traverse, the robot updates the map itself according to SLAM algorithm. An illustration is shown in figure 3. As a result, the path gets updated.

\section{Experiment}

We tested the accuracy of the robot in bright (55 lux - 60 lux) and dark (15 lux 20 lux) lighting conditions. System was tested using five users located to the sides of the unstructured room of dimension $5.7 \mathrm{~m} \times 5.6 \mathrm{~m} \times 3.3 \mathrm{~m}$ And named each user 1 to 5 and assigned coordinates on map. We created a Graphical User Interface (GUI) which is shown in the figure 5. It was built using RESTful API and Python programming language. It is installable in existing computers of users. When a user provides a name by typing, the pre-assigned coordinate (table 1) is passed to Rviz view node in Centre location PC. Rviz view node then sets the goal location and sends the minimal path to the robot. Robot then follows the path and reaches the goal

\begin{tabular}{|l|}
\hline \multicolumn{2}{|c|}{ Name : } \\
\hline \multicolumn{1}{|c|}{ Go to the Place } \\
\hline \multicolumn{1}{|c|}{ Gorimary win } \\
\hline $\begin{array}{l}\text { Isanka } \\
\text { Chamalie } \\
\text { Chathu } \\
\text { Tharaka } \\
\text { Isanka } \\
\text { Sathya } \\
\text { Tharaka } \\
\text { Chamalie } \\
\text { Sathya } \\
\text { Chathu }\end{array}$ \\
\hline
\end{tabular}

Figure 5. Graphical User Interface program for robot controlling

location. We monitored the success rate of completion of this process for each user by conducting the experiment randomly for 100 times per user in each lighting condition.

\section{RESULTS \& DISCUSSION}

The experimental result shows more than $98 \%$ accuracy of achieving goal on average according to the user commands. It gives accuracy of $100 \%$ for three of the selected users in both dark and bright illumination conditions. It could be seen that $98.8 \%$ of accuracy in dark room, and the robot has lost its way only once among a total of 500 rounds of navigation. Further, it has completed goals in 490 times out of 500 total rounds in bright lighting condition that equals to 98\% accuracy. Results are shown in the figure 6 . It should be noted that the robot uses IR image to measure the distance to objects in the environment. This feature is included in Primesense.

\section{CONCLUSIONS \& FURTHER WORK}

Localization and mapping are the two main features of a movable robot. In order to navigate safely for a target location, 
avoiding obstacles in an unstructured environment according to the user commands is a challenging task. We have built an office assistant robot system having three units that communicate wirelessly. A separate processing unit, a movable robot structure and robot controlling unit were used in advance. We trained our system in an unstructured indoor environment and tested its accuracy in bright and dark illumination conditions. The experiment results prove that the system we have implemented has high accuracy and it can be successfully used in an unstructured room and that the robot will help to make people's tasks easier. Further, the results indicate that the accuracy is independent of the lighting condition of the environment, because the robot capture environment using IR camera.

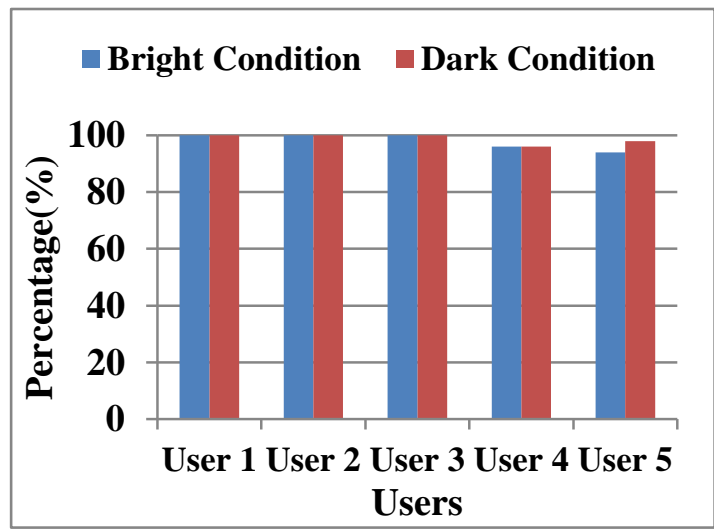

Figure 6. Accuracy of office assistant robot system according to the user commands in both bright and dark lighting conditions

The developed robot system will be further improved in the near future to be navigated according to voice commands made through the mobile phone.

\section{REFERENCES}

Adikari, A.M.S.B., Ganegoda, N.G.C. and Wanniarachchi, W.K.I.L., 2017. Noncontact human body parameter measurement based on kinect sensor. IOSR Journal of Computer Engineering, 19(3), pp.80-85..

An, Z., Hao, L., Liu, Y. and Dai, L., 2016. Development of Mobile Robot SLAM Based on ROS. International Journal of Mechanical Engineering and Robotics Research, 5(1), pp.47-51.

Chen, L., Wang, S., Hu, H., Gu, D. and Dukes, I., 2008. Voice-directed autonomous navigation of a smartwheelchair. In Smart Wheelchairs and Brain-Computer Interfaces (pp. 405-424). Academic Press.

Correa, D.S.O., Sciotti, D.F., Prado, M.G., Sales, D.O., Wolf, D.F. and Osorio, F.S., 2012, May. Mobile robots navigation in indoor environments using kinect sensor. In 2012 Second Brazilian Conference on Critical Embedded Systems (pp. 36-41). IEEE.

Diddeniya, S.I.A.P., Rathnayake, K.A.S.V., Gunasinghe, H.N. and Wanniarachchi, W.K.I.L., 2017. Integrating irobot-arduino-kinect systems: An educational voice and keyboard operated wireless mobile robot. International Journal of Scientific Engineering and Technology, 6(6), pp.188-192.

Elfes, A., 1987. Sonar-based real-world mapping and navigation. IEEE Journal on Robotics and Automation,3(3), pp.249265.

Gao, R., Zhang, L. and Zhang, S., 2017, September. Research on Mobile Robot SLAM Based on Laser Range Finder. In The 7th International Conference on Computer Engineering and 
Networks (Vol. 299, p. 016). SISSA Medialab.

Hamzeh, O. and Elnagar, A., 2015, December. A Kinect-based indoor mobile robot localization. In 2015 10th International Symposium on Mechatronics and its Applications (ISMA) (pp. 1-6). IEEE.

Hiemstra, P. and Nederveen, A., 2007. Monte carlo localization.

Ioakeimidis, P.A., 2018. Integration of ROS, Turtlebot, RPLIDAR, RFID technologies and algorithm implementation for navigation and RFID tag detection in a warehouse.

Li, Z., Xiong, Y. and Zhou, L., 2017, December. ROS-Based Indoor Autonomous Exploration and Navigation Wheelchair. In 2017 10th International Symposium on Computational Intelligence and Design (ISCID) (Vol. 2, pp. 132-135). IEEE.

Rathnayake, K.A.S.V., Diddeniya, S.I.A.P., Wanniarachchi, W.L., Nanayakkara, W.H.K.P. and Gunasinghe, H.N., 2016, December. Voice operated home automation system based on Kinect sensor. In 2016 IEEE International Conference on Information and Automation for Sustainability (ICIAfS) (pp. 1-5). IEEE.

Rathnayake, K.A.S.V., Wanniarachchi, W.K.I.L. and Nanavakkara, W.H.K.P., 2018, April. Human computer interaction system for impaired people by using kinect motion sensor: Voice and gesture integrated smart home. In 2018 Second International Conference on Inventive Communication and Computational Technologies (ICICCT) (pp. 531-536). IEEE.

Sherwin, T., Easte, M., Chen, A., Wang, K. and Dai, W., 2018. A Single RF Emitter-Based Indoor Navigation Method for Autonomous Service Robots. Sensors, 18(2), p.585.

Whyte, H. D. J. R. \& Magazine, A. 2006. Simultaneous localisation and mapping (SLAM): Part I the essential algorithms.

Xu, Q., Zhao, J., Zhang, C. and He, F., 2015, August. Design and implementation of an ROS based autonomous navigation system. In 2015 IEEE International Conference on Mechatronics and Automation (ICMA) (pp. 2220-2225). IEEE.

Zaman, S., Slany, W. and Steinbauer, G., 2011, April. ROS-based mapping, localization and autonomous navigation using a Pioneer 3-DX robot and their relevant issues. In 2011 Saudi International Electronics, Communications and Photonics Conference (SIECPC) (pp. 1-5). IEEE.

Zhang, Z., 2012. Microsoft kinect sensor and its effect. IEEE multimedia, 19(2), pp.4-10.

Zhou, C., Wei, Y. and Tan, T., 2003, September. Mobile robot self-localization based on global visual appearance features. In 2003 IEEE International Conference on Robotics and Automation (Cat. No. 03CH37422) (Vol. 1, pp. 12711276). IEEE..

\section{ACKNOWLEDGMENT}

Authors wish to acknowledge University of Sri Jayewardenepura (Grant No ASP/01/RE/SCI/2017/13) for the funding support and to Mr. O. K. D. M. Priyantha, Workshop Technical Officer, Department of Physics, Faculty of Applied Sciences, University of Sri Jayewardenepura for the given support. Additionally, a sincere gratitude is forwarded to the volunteers who have supported to test this system. 